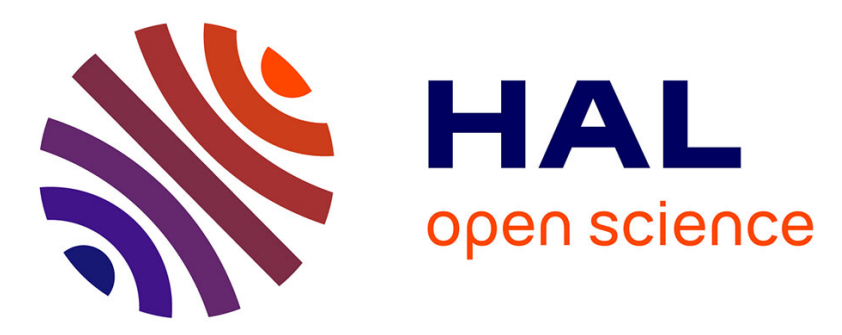

\title{
Energy predictions of turbulent boundary layer induced mid-high frequency structural vibrations
}

Mohamed Ichchou, Olivier Bareille, Yves Jacques

\section{To cite this version:}

Mohamed Ichchou, Olivier Bareille, Yves Jacques. Energy predictions of turbulent boundary layer induced mid-high frequency structural vibrations. Journal of Wind Engineering and Industrial Aerodynamics, 2009, 97, pp.63-76. 10.1016/j.jweia.2008.11.001 . hal-01011722

\section{HAL Id: hal-01011722 \\ https://hal.science/hal-01011722}

Submitted on 26 Jun 2014

HAL is a multi-disciplinary open access archive for the deposit and dissemination of scientific research documents, whether they are published or not. The documents may come from teaching and research institutions in France or abroad, or from public or private research centers.
L'archive ouverte pluridisciplinaire $\mathbf{H A L}$, est destinée au dépôt et à la diffusion de documents scientifiques de niveau recherche, publiés ou non, émanant des établissements d'enseignement et de recherche français ou étrangers, des laboratoires publics ou privés. 


\section{ENERGY PREDICTIONS OF TURBULENT BOUNDARY LAYER INDUCED MID-HIGH FREQUENCY STRUCTURAL VIBRATIONS}

BY

M.N. ICHCHOU, O. BAREILLE and Y. JACQUES

Laboratoire de Tribologie et de Dynamique des Systèmes, UMR CNRS 5513, Ecole Centrale de Lyon

$\begin{array}{llc}\text { Number of pages } & : & 41 \\ \text { Number of tables } & : & 1 \\ \text { Number of Figures } & : & 19\end{array}$

Short running headline: Energy modelling of TBL-induced vibration

Keywords: Turbulent Boundary Layer, Bending vibration, Energy flow methods, Mid-High frequencies. 


\section{ENERGY PREDICTIONS OF TURBULENT BOUNDARY LAYER INDUCED MID-HIGH FREQUENCY STRUCTURAL VIBRATIONS}

BY

M.N. ICHCHOU, O. BAREILLE and Y. JACQUES

POSTAL ADDRESS AND CORRESPONDING AUTHOR:

\section{Prof M. ICHCHOU}

Laboratoire de Tribologie et Dynamique des Systèmes

Equipe Dynamique des Structures et des Systèmes,

Ecole Centrale de Lyon, Bâtiment E6

36 avenue Guy de Collongue, 69130 Ecully, France.

$$
\begin{aligned}
& \text { tel : } 0472186230 \\
& \text { fax : } 0472189144
\end{aligned}
$$

e-mail : mohamed.ichchou@ec-lyon.fr 


\begin{abstract}
This paper addresses the study of vibrations induced by turbulent boundary layers flowing over plate-like structures. The aim is here to propose a predictive method in order to evaluate the vibroacoustical behaviour of a plate excited by turbulent boundary layer pressure fluctuations. The ultimate goal is to develop tools for predicting internal cabin noise in an aircraft during flight. Hence turbulent boundary layer (TBL) is considered. Different TBL models are discussed and compared. A prediction method well suited for mid-high frequencies is also presented. The method called, Simplified Energy Method (SEM), is based on local energetic considerations and extends the well known Statistical Energy Analysis (SEA). Two different SEM formulations are presented. The first one is of heat conduction type, whilst the second is radiative. The TBL source model was integrated in the local energy proposed formulations. When applied to a simple Kirchhoff-Love simply supported plate, SEM allows prediction of averaged energy quantities. Comparisons are drawn between heat and radiative formulations. Good concordance was observed between energy results and pure analytical calculations of the structural response under TBL excitation over a wide frequency range.
\end{abstract}




\section{INTRODUCTION}

With increasing flight speed and the generalisation of air transportation has appeared a necessity to reduce structural fatigue for aircraft and acoustic/vibration levels for their payload and passengers. Passengers' comfort, during the past years, has become one of the principal concerns of various actors in the aeronautic industry, since it is actually one of the essential demands of users. Among the factors that are considered to affect the wellbeing of passengers, acoustics and vibrations are ranked at the top of the list. Interior noise related to the external flow has become a significant noise contributor under certain conditions even for ground transportation vehicles.

Turbulent external flow related loads are principally of two types, involving two areas of research. First, turbulent fluctuations behave as acoustic sources. The acoustic field resulting from turbulence loads the panels which, in turn, transmit noise inside the vehicle. These phenomena are qualified as aero-acoustic. At low Mach numbers, they become important only in the presence of a protruding accessory that induces strong turbulent stresses in a flow region that consequently behaves as an acoustic source.

Fig. 1: Structural vibration due to Turbulent Boundary Layer

Second, the interaction between turbulent flow and external panels causes wall pressure fluctuations that generate panel vibrations, which radiate sound into the passenger or payload compartment (see Fig. 1). In all cases, fluid inertia forces will become greater than viscous forces and a turbulent boundary layer (TBL) develops if velocity is high enough and if the structure is large.

On the one hand, the numerical prediction of such phenomena is costly when processed through conventional methods such as Finite-Element based ones. This is especially true for distributed random excitation, such as TBL excitation, for which the forced response problem requires exponentially more computational effort than the modal analysis of the structure. Some alternative methods, still displacement based, like the dynamic stiffness method and the spectral FEM, (Birgersson et al 2003) succeeded in reducing the number of degrees of freedom and increase accuracy. The elements are formulated and assembled as in the standard FEM while the basis 
functions are exact solutions to the equations of motion. Normally, the spectral FEM, and the dynamic stiffness method (DSM), consider excitation at the element ends only. Though the above method is efficient and accurate, the relevant information regarding the response of the structure rely on an averaged value. This true to energy based method, to which cross-spectral densities and cross-correlation functions can be related in a straight forward manner. The weakness of energy methods is in the models used for injected power. Provided the TBL excitation can be well described, one can expect some good results. The most common TBL model is attributed to Corcos (Corcos 1967), and has been validated experimentally. Yet, it overestimates the wall-pressure crossspectral density at wave-numbers below the convective peak. Some improved models were therefore developed to give a better representation of the sound source associated to the TBL interacting with surface panels (Zheng 2003). It should be pointed out that the weak coupling assumption (Graham 1997) is accepted in the current study. This assumption corresponds to the assumption, implicit in acoustic analogy analyses, that the basic turbulence structure is essentially unaffected by the acoustic motions. Feedback from the vibrating panel can affect the properties of turbulent flow and its pressure loading. However, when trying to predict the body panel responses, the complexity is such that most studies keep a weak interaction hypothesis, i.e., they neglect panel feedback on flow. This approach, when valid, i.e., without aeroelastic instabilities, allows computation of the panel response from the properties of the wall pressure field experimentally determined on a hard surface. The predictions obtained from the weak coupling approximation have been shown to work well in a wide range of cases (Graham 1997), except for the differences between the theoretical results and measured data mentioned in (Blake 1986) in supersonic flow cases.

On the other hand, TBL excitation is a typical wide frequency band excitation. It should thus be noted that well established and detailed numerical methods like finite element or boundary equation based formulations run into important limitations as frequency increases. Specific medium and high frequency range methods are then needed to deal with the vibrational behaviour in this particular frequency range. Among possible alternatives, two different philosophies can be found in the specialised literature. The first class of analysis methods are based on displacement variables. These methods tend to reach the medium and high frequency domain using both phase and amplitude behaviour. Among these methods we note the work done by C. Soize (Soize 1993) who introduced the structural-acoustic fuzzy theory. Ultimately, the complex ray theory developed by Ladeveze (Ladeveze 1996) can also be considered as displacement approach to medium and high frequencies. The second class of analysis methods are based on energy variables. The Statistical Energy 
Analysis (SEA) (Lyon 1975) is the recognized originator. SEA gives the mechanical energy of complex built-up structures. Statistical Energy Analysis offers an alternative form of vibrational and acoustical state. The statistical aspects included are mainly related to the high frequency features. Modelling uncertainties made deterministic approaches often irrelevant at high frequency. SEA also provides a way of energy transfer analysis. Stored energies, dissipated energies, exchanged powers as well as injected powers, are the main variables. SEA tends to characterise the amplitude of vibrational signals, without any information about phase. This is a major simplification. It offers reduced computational costs, mainly at high frequencies. However SEA suffers from its axiomatic formulation and still requires improvements, as indicated in (Fahy 1994) where an interesting survey and a number of critical comments are given. Beyond these studies, a number of works attempt to enhance the Statistical Energy Analysis robustness and predictivity. Among these attempts we may cite the earlier work of Nefske and Sung (Nefske and Sung 1987) who proposed the use of an energy diffusion model to predict the space spread of energy density within subsystems. Basically, this way of thinking can be viewed as a local energy formalism whereas the SEA formalism is based on global energies of finite subsystems. This model has been improved by Bernhard and his colleagues (Wohlever and Bernhard 1992, Han et al. 1999). Among other contributors to this subject, we may mention (Langley 1995, Carcatera and Sestieri, 1995) where interesting discussions are given. Developments at the Ecole Centrale de Lyon in the context of energy models started with the so-called general energy method (Ichchou 1996). The main goal of this method was to reformulate the classical displacement models using four energy variables: the total energy as well as the Lagrangian energy density, the active and the reactive energy flow. Eliminating the Lagrangian energy density and the reactive energy flow, the general energy formulation leads to an interesting energy model well suited for medium and high frequency dynamics. In fact, from a propagative approach, it has been shown that the Lagrangian energy density and the reactive energy flow is mainly linked to wave interferences or singularities. So that, taking into account only the incoherent contribution of waves to the energy variables leads to the formulation of the local energy approach discussed here and called Simplified Energy Method (SEM).

Two main energy formulations have been formulated. The first one is similar to the heat transfer equation and can be solved through finite element energy based techniques (among others). The second formulation is similar to radiative equations and can be solved through boundary element energy based approaches. The main goal of this paper is to synthesise these formulations in order to show the interest of this local energy approach in the context of predicting TBL induced vibration. 
This paper deals with such predictions for the vibroacoustical behaviour of a plate-like structure by means of energy based approaches. These latter are first introduced in section 2, as an alternative to conventional SEA (Statistical Energy Analysis). In section 3, an overview of the existing TBL (Turbulent Boundary Layer) models is given. The choice of one of these is then justified. Section 5 presents the numerical compared results obtained by means of the two energy approaches in an example fully described in section 4.

\section{LOCAL ENERGY APPROACH FORMULATIONS}

The main goal of this section is to discuss local energy approach formulations. Dynamical equations of motion of structures will then be expressed in terms of energy. This leads to practical efficient methods especially for high and mid frequency vibrations. We start with a reminder of basic equations of energy conservation in general elastoacoustics. We then formulate the problem in terms of energy flow balance. This is the first step in the local energy as well as global SEA formulation. Then, using a wave approach very similar to the one given in (Ichchou and Jezequel 1996) in the steady state case, smooth energy behaviour is studied for damped structures. This wave approach is combined with statistical representation, in order to reach averaged energy levels for complex dynamical situations. Specifically, the analysis starts with elementary wave energy behaviour. In this context, the discussion starts with a plane wave assumption, leading to a differential energy equation of the associated energy parameters. After that, spherical waves are considered and related energy equations are given. The transition, from elementary waves to a complex waves composed dynamics is then discussed. A fundamental set of required assumptions are thus introduced and commented. The main assumption is related to the non-coherence situation between waves, which leads to a local superposition principle. Finally, a foundation of the local energy approach is given.

\subsection{PRELIMINARIES}

In view of describing the energy transfer inside the medium, two continuous energy fields are introduced. The first energy quantity is merely the total energy density $W(\overrightarrow{\boldsymbol{x}}, t)$ defined as the sum of the potential energy density and the kinetic energy density. $\overrightarrow{\boldsymbol{x}}$ being an abscissa point of the studied medium. The second energy variable $\overrightarrow{\mathbf{I}}(\overrightarrow{\boldsymbol{x}}, t)$ is the energy flow. These quantities are local, in contrast with energies per subsystem involved in SEA. The energy balance equation from 
continuum mechanics, obeying the conservation principle, and which govern the energy density in various vibrating systems can be described by:

$\frac{\partial W}{\partial t}(\overrightarrow{\boldsymbol{x}}, t)+\operatorname{div}(\overrightarrow{\mathbf{I}}(\overrightarrow{\boldsymbol{x}}, t))+p_{d i s s}=p_{i n j}$

where div is the divergence operator, $p_{\text {diss }}$ is the dissipated power density. $p_{i n j}$ is connected to exterior forces that will inject power in the system. The damping model adopted here is the same as in SEA (power density being dissipated is proportional to the energy density). Hence:

$p_{\text {diss }}(\overrightarrow{\boldsymbol{x}}, t)=\eta \omega W(\overrightarrow{\boldsymbol{x}}, t)$

where $\eta$ is the damping loss factor and $\omega$ the circular frequency. The validity of this relationship has been discussed in the literature (Lyon 1975) about SEA. In order to derive the energy density equations, a wave description of vibrational-acoustical behaviour is adopted. In the subsequent presentation, planes and symmetrical propagating disturbances in an elastic and dissipative medium are considered. Each propagating wave involves partial energy quantities defined as the energy variables associated to those waves. In order to establish a relationship between the partial energies $W_{i}(\overrightarrow{\boldsymbol{x}}, t)$ and $\overrightarrow{\mathbf{I}}_{i}(\overrightarrow{\boldsymbol{x}}, t)$ and the total ones $W(\overrightarrow{\boldsymbol{x}}, t)$ and $\overrightarrow{\mathbf{I}}(\overrightarrow{\boldsymbol{x}}, t)$, an additional assumption is introduced. In fact, a superposition principle is assumed and applied here for all quadratic variables: $\wp=\sum_{i} \wp_{i}$

$\wp, \wp_{i}$ being any quadratic variable, representing either a partial energy or a global energy associated with a particular wave field. This assumption is often used in statistical phenomena in physics. In fact, we can find such an assumption in room acoustics, electromagnetism, etc. This assumption is typical in the high frequency literature (see (Lyon 1975) and (Sestieri and Carcaterra 2003) for further details). In the following developments, an intrinsic energy law will be used. This equation is often introduced in order to define the wave velocity $c_{i}$. Where:

$\overrightarrow{\mathbf{I}}_{i}(\overrightarrow{\boldsymbol{x}}, t)=c_{i} W_{i}(\overrightarrow{\boldsymbol{x}}, t) \overrightarrow{\boldsymbol{n}}_{i}$

This expression was shown to be valid for conservative wave fields. It will be used in the following as a first step in the energy density equation process for lightly damped structures.

\subsection{ELEMENTARY WAVE ANALYSIS: PLANE and SPHERICAL WAVES}

In (Ichchou and Jezequel 1996, Ichchou et al. 2001), it was shown that, considering a plane wave in an isolated medium, as a particular solution of the motion equation, it verifies the energy equation: 
$-c_{i}^{2} \Delta W_{i}+\frac{\partial^{2} W_{i}}{\partial t^{2}}+2 \eta \omega \frac{\partial W_{i}}{\partial t}+(\eta \omega)^{2} W_{i}=0$

Under continuous forced harmonic excitation (angular frequency $\omega$ ), through time averaging, the following equation is obtained:

$-c_{i}^{2} \Delta W_{i}+(\eta \omega)^{2} W_{i}=0$

Or equivalently through linearity in a forced medium

$-c_{i}^{2} \Delta W_{i}+(\eta \omega)^{2} W_{i}=\eta \omega p_{i n j}$

This equation is equivalent to the equation of heat diffusion with a term of convection. This equation governs the energy density changes. It provides a space average of the predicted energy density. This equation has been commented and discussed in-depth by many authors (see for instance (Langley 1995) and (Ichchou 1996) for further details). Let us consider now symmetrical wave fields. Specifically, we will consider cylindrical waves in dimension 2, and spherical waves in dimension 3. In this case, due to the revolution symmetry, the energy variables are $W_{i}(r, t)$ and $\overrightarrow{\mathbf{I}}_{i}(r, t)$, where $\mathrm{r}$ is the radial coordinate. Performing the same demonstration as for plane waves, the following equation is readily obtained:

$$
-c_{i}^{2} \frac{1}{r^{n-1}} \frac{\partial^{2}\left(r^{n-1} W_{i}\right)}{\partial r^{2}}+\frac{\partial^{2} W_{i}}{\partial t^{2}}+2 \eta \omega \frac{\partial W_{i}}{\partial t}+(\eta \omega)^{2} W_{i}=0
$$

We can also study the harmonic stationary case, whose equation is:

$$
-c_{i}^{2} \frac{1}{r^{n-1}} \frac{\partial^{2}\left(r^{n-1} W_{i}\right)}{\partial r^{2}}+(\eta \omega)^{2} W_{i}=0
$$

We notice that this equation is distinct from the equation of heat diffusion. It correctly gives the energy behaviour of infinite systems.

\subsection{ENERGY FIELDS RECONSTRUCTION: HEAT CONDUCTION EQUATION}

From the local energy equations of elementary fields, we will be able to derive the local energy equations for more complex wave fields. Hence, for one dimension, the knowledge of the wave content will lead to the formulation of the problem in terms of the global field. The situation is more complicated for two or three dimension where the wave contents and nature can be of various kinds. New assumptions are then needed to deal with multi-dimensional cases, like for instance, vibrating Kirchhoff-Love plates. 
Fig. 2: Energy field reconstruction with plane waves in complex 2 and 3 dimensional media: a reverberant field is considered

Fig. 3: Energy field reconstruction with symmetrical waves in complex 2 and 3 dimensional media: direct and reverberant fields are indicated

Fig. 4: Energy field reconstruction with symmetrical waves in complex 2 and 3 dimensional media: primary and secondary sources definitions

Fig. 2 and 3 show two distinct ways of energy reconstruction. In both techniques, energy variables are defined as the superposition of a direct field and a reverberant field. In both techniques, the direct field is calculated assuming a symmetrical wave field as described above. The difference lies in the reverberant field treatment. In Fig. 2 the reverberant field is assumed to be a set of plane waves. This approach leads to a simple differential operator on the reverberant energy density. The second solution considers the reverberant field as the result of secondary sources located at boundaries as shown in Fig. 4. So, only symmetrical wave fields are considered. This solution was first proposed by Kuttruff (Kuttruff 1997). It is also known as the radiosity method. Further details can be found in (Schmitt 2004). Both formulations are explained below. Some indications about their numerical resolution are also briefly given.

\subsection{ENERGY FIELDS RECONSTRUCTION: HEAT CONDUCTION EQUATION}

Let us consider a medium in which only plane waves exist. The medium is assumed to be homogeneous and isotropic, so that all the waves have the same energy velocity, noted for convenience as, $\mathrm{c}_{\mathrm{g}}$ and which is a physical time and space constant. It was shown that the energy variables, associated to the considered field obeys:

$-c_{g}^{2} \Delta(W)+\frac{\partial^{2} W}{\partial t^{2}}+2 \eta \omega \frac{\partial W}{\partial t}+(\eta \omega)^{2} W=0$

In the case where the considered medium is defined by a distribution of exterior forces, this will be represented by a right-hand side $p_{i n j}$. Under harmonic stationary excitation, this equation becomes simply:

$-c_{g}^{2} \Delta(W(\overrightarrow{\boldsymbol{x}}, t))+(\eta \omega)^{2} W(\overrightarrow{\boldsymbol{x}}, t)=\eta \omega p_{i n j}$

It is a heat conduction type equation with a term of convection. Numerical resolution of this equation requires three main parameters. The first one is the energy group velocity. This parameter is known for most conventional structural elements (see reference (Ichchou and Jezequel 1996) for 
details). The second parameter is the damping loss factor which is assumed to be given and finally the power injected density which is directly related to the source. Calculation of this quantity in the context of TBL excitation is explained below. It should be also pointed out, that Eq 11 needs boundary conditions to be given in terms of energy variables. Detailed discussions of these boundary conditions can be found in many references (see for instance (Ichchou 1996)). In the case considered in this paper, the plate is assumed to be boundary conservative (non absorbing). This condition is found in many classic mechanical situations (clamped, free, pinned,...). So that the non absorbing boundary condition can be simply expressed in terms of energy flow such as:

$$
\frac{\partial W}{\partial \overrightarrow{\boldsymbol{n}}_{\text {ext }}}=0
$$

$\overrightarrow{\boldsymbol{n}}_{\text {ext }}$ being the external normal vector to the considered boundary. Eq 11 and 12 can finally be solved within a finite element energy based context, once all the relevant quantities are provided.

\subsection{ENERGY FIELDS RECONSTRUCTION: RADIATIVE TRANSFER EQUATION}

Let us assume that only spherical waves can be propagated in the studied medium, with an energy velocity $\mathrm{c}_{\mathrm{g}}$. In this case the energy equation derived above also stands for the global energy and so that for stationary dynamics:

$$
-c_{g}^{2} \frac{1}{r^{n-1}} \frac{\partial^{2}\left(r^{n-1} W\right)}{\partial r^{2}}+(\eta \omega)^{2} W=0
$$

Let us note $G(S, M)$ the impulsional solution corresponding to this equation, and $\overrightarrow{\mathbf{H}}(S, M)$ the associated intensity vector. $\mathrm{S}$ will designate below the source point, and $\mathrm{M}$ the point where the energy density is evaluated. Analytical expressions of both functions are, in two dimensional space, given by:

$G(S, M)=\frac{e^{-\frac{\eta \omega S M}{c_{g}}}}{S M}$, and $\overrightarrow{\boldsymbol{H}}(S, M)=\frac{e^{-\frac{\eta \omega S M}{c_{g}}}}{S M^{2}} \overrightarrow{\boldsymbol{M}}$

The destructive interferences assumption allows one to build the solution by superposition of energies associated respectively to direct and reverberated fields (see Fig. 3 and 4). So, at each point of the domain $\Omega$, the contributions of real sources (corresponding to external forces) and fictive unknown sources (located on the boundary $\partial \Omega$ ) are summed. Both contributions are detailed in the following expression:

$$
\begin{gathered}
\forall S \in \Omega, \quad \rho(S) G(S, M) \quad \text { and } \quad \rho(S) \overrightarrow{\boldsymbol{H}}(S, M) \\
\forall P \in \partial \Omega, \quad \sigma(P) f\left(\overrightarrow{\boldsymbol{n}}_{M P} \cdot \overrightarrow{\boldsymbol{n}}_{P}\right) G(P, M) \quad \text { and } \quad \sigma(P) f\left(\overrightarrow{\boldsymbol{n}}_{M P} \cdot \overrightarrow{\boldsymbol{n}}_{P}\right) \overrightarrow{\boldsymbol{H}}(P, M)
\end{gathered}
$$


$f$ is a scalar function, which depends on the angle between the direction of active intensity and the boundary external normal. $M$ is the point of the considered domain, the primary source density is $\rho(S)$ over $\Omega$, and the primary sources are proportional to the injected power density $p_{i n j}$. Here, $f$ follows a Lambert directivity law, so that the total energy density and the active intensity somewhere in the considered media can be written simply as:

$$
\begin{aligned}
& W(M)=\iint_{\Omega} \rho(S) G(S, M) d \Omega+\int_{\partial \Omega} \sigma(P)\left(\overrightarrow{\boldsymbol{n}}_{M P} \cdot \overrightarrow{\boldsymbol{n}}_{P}\right) G(P, M) d P \\
& \overrightarrow{\boldsymbol{I}}(M)=\iint_{\Omega} \rho(S) \overrightarrow{\boldsymbol{H}}(S, M) d \Omega+\int_{\partial \Omega} \sigma(P)\left(\overrightarrow{\boldsymbol{n}}_{M P} \cdot \overrightarrow{\boldsymbol{n}}_{P}\right) \overrightarrow{\boldsymbol{H}}(P, M) d P
\end{aligned}
$$

The injected power density in the system is equal to $2 \rho \pi c_{g}$ because the energy density and the active intensity vector verify the following equation:

$\operatorname{div} \overrightarrow{\boldsymbol{I}}+\eta \omega W=2 \rho \pi c_{g}$

To complete this description, a boundary condition is considered in terms of energy. Indeed, if conservative boundaries are assumed then no energy is flowing from the boundary (the scalar product of the active intensity and of the exterior normal is null). Applying this result to the previous expression of active intensity, a Freedholm integral type equation of the second kind is shown as:

$$
2 c_{g} \sigma(P)=\left(\iint_{\Omega} \rho(S) \overrightarrow{\boldsymbol{H}}(S, P) d \Omega+\int_{\partial \Omega} * \sigma\left(P^{\prime}\right)\left(\overrightarrow{\boldsymbol{n}}_{P P^{\prime}} \cdot \overrightarrow{\boldsymbol{n}}_{P^{\prime}}\right) \overrightarrow{\boldsymbol{H}}\left(P^{\prime}, P\right) d P^{\prime}\right) \cdot \overrightarrow{\boldsymbol{n}}_{P}
$$

The resolution of this integral equation is obtained by discretising the boundary in $\mathrm{n}$ segments $\mathrm{S}_{\mathrm{k}}$. $\mathrm{P}_{\mathrm{k}}$ is the middle of the segment $\mathrm{S}_{\mathrm{k}}$ and $\mathrm{Q}$ is a variable point on the segment. The source density is assumed to be constant on each segment and takes the value $\sigma_{\mathrm{k}} \cdot \overrightarrow{\boldsymbol{n}}_{k}$ is the exterior normal vector to the domain at $\mathrm{P}_{\mathrm{k}}$. The equation becomes:

$$
2 c_{g} \sigma_{k}=\iint_{\Omega} \rho(S) \overrightarrow{\boldsymbol{H}}\left(S, P_{k}\right) d \Omega \cdot \overrightarrow{\boldsymbol{n}}_{k}+\sum_{\substack{l=1 \\ l \neq k}}^{n} \sigma_{l} \int_{S_{l}}\left(\overrightarrow{\boldsymbol{n}}_{P_{k} Q} \cdot \overrightarrow{\boldsymbol{n}}_{Q}\right)\left(\overrightarrow{\boldsymbol{H}}\left(Q, P_{k}\right) \cdot \overrightarrow{\boldsymbol{n}}_{k}\right) d Q
$$

The $\mathrm{n}$ values of $\sigma$ for a type of wave are obtained in solving the preceding system. After their evaluation, one can obtain the fields of energy density and active intensity in the domain $\Omega$ :

$$
\begin{aligned}
& W(M)=\iint_{\Omega} \rho(S) G(S, M) d \Omega++\sum_{l=1}^{n} \sigma_{l} \int_{S_{l}}\left(\overrightarrow{\boldsymbol{n}}_{M Q} \cdot \overrightarrow{\boldsymbol{n}}_{Q}\right) G(Q, M) d Q \\
& \overrightarrow{\boldsymbol{I}}(M)=\iint_{\Omega} \rho(S) \overrightarrow{\boldsymbol{H}}(S, M) d \Omega+\sum_{l=1}^{n} \sigma_{l} \int_{S_{l}}\left(\overrightarrow{\boldsymbol{n}}_{M Q} \cdot \overrightarrow{\boldsymbol{n}}_{Q}\right) \overrightarrow{\boldsymbol{H}}(Q, M) d Q
\end{aligned}
$$

In the multidimensional domain, Kuttruff proposed (Kuttruff 1997) an integral equivalent to the one provided here. This method is known as the "radiosity method". It was principally applied in the domain of room acoustics. Further discussions of this formulation can be found in (Schmitt 2004). 